\title{
Effects of aleglitazar, a balanced dual peroxisome proliferator-activated receptor $\alpha / \gamma$ agonist on glycemic and lipid parameters in a primate model of the metabolic syndrome
}

\author{
Barbara C Hansen ${ }^{1 *}$, Xenia T Tigno ${ }^{2}$, Agnes Bénardeau ${ }^{3}$, Markus Meyer ${ }^{3}$, Elena Sebokova ${ }^{3}$, Jacques Mizrahi ${ }^{3}$
}

\begin{abstract}
Background: Glycemic control and management of dyslipidemia to reduce cardiovascular risk are major therapeutic goals in individuals with type 2 diabetes mellitus (T2DM). This study was performed to evaluate the effects of aleglitazar, a balanced dual peroxisome proliferator-activated receptor $\alpha / \gamma$ (PPAR $\alpha / \gamma)$ agonist, on both lipid and glycemic parameters in obese, hypertriglyceridemic, insulin-resistant rhesus monkeys.
\end{abstract}

Methods: A 135-day efficacy study was performed in six rhesus monkeys. After a 28-day baseline assessment (vehicle only), monkeys received oral aleglitazar $0.03 \mathrm{mg} / \mathrm{kg}$ per day for 42 days, followed by a 63-day washout period. Plasma levels of markers of glycemic and lipid regulation were measured at baseline, at the end of the dosing period, and at the end of the washout period.

Results: Compared with baseline values, aleglitazar $0.03 \mathrm{mg} / \mathrm{kg}$ per day reduced triglyceride levels by an average of $89 \%$ (328 to $36 \mathrm{mg} / \mathrm{dL} ; \mathrm{P}=0.0035$ when normalized for baseline levels) and increased high-density lipoprotein cholesterol levels by $125 \%$ (46 to $102 \mathrm{mg} / \mathrm{dL} ; \mathrm{P}=0.0007$ ). Furthermore, aleglitazar reduced low-density lipoprotein cholesterol levels (41\%) and increased levels of apolipoprotein A-I (17\%) and A-II (17\%). Aleglitazar also improved insulin sensitivity by $60 \%(P=0.001)$. Mean body weight was reduced by $5.9 \%$ from baseline values with aleglitazar at this dose $(P=0.043)$.

Conclusions: Aleglitazar, a dual PPAR $\alpha / \gamma$ agonist, has beneficial effects on both lipid and glucose parameters and may have a therapeutic role in modifying cardiovascular risk factors and improving glycemic control in patients with T2DM.

\section{Introduction}

According to the World Health Organization, cardiovascular disease accounts for half of all deaths in individuals with type 2 diabetes mellitus (T2DM) [1]. This highlights the need to reduce the cardiovascular risk in these individuals by management of the major T2DMrelated risk factors: dyslipidemia, hyperglycemia, hypertension, and body weight $[2,3]$. Dyslipidemia in subjects with T2DM is characterized by elevated levels of triglycerides (TGs) and reduced levels of high-density

\footnotetext{
* Correspondence: bchansen@aol.com

'Department of Internal Medicine and Pediatrics, University of South Florida, Tampa, FL, USA

Full list of author information is available at the end of the article
}

lipoprotein cholesterol (HDL-C). Although levels of low-density lipoprotein cholesterol (LDL-C) are not significantly increased, the profile in T2DM is characterized by atherogenic small dense particles. Statin therapy has been proven to reduce effectively plasma levels of LDL-C; however, effects on HDL-C and TG levels are more modest. Therefore, a safe and efficacious therapy that can provide improvements in the overall lipid profile of patients with T2DM, while achieving good glycemic control, would have great value in reducing the risk of cardiovascular disease.

Research has shown that obesity-associated T2DM that develops spontaneously under natural and "healthy" diet conditions during adulthood in several non-human 
primate species, such as the rhesus monkey (Macaca mulatta), closely parallels the disease affecting humans [4-10]. Evidence from longitudinal studies has also provided thorough insight into the development of T2DM in rhesus monkeys $[6,11]$. Furthermore, the profile of naturally occurring dyslipidemia, despite a low-fat diet, closely reflects the lipid profiles of humans [12]. As such, these primates are a well-recognized, spontaneously developing animal model for the metabolic syndrome and T2DM in humans, and provide an excellent preclinical screening tool for novel antidiabetic agents [13-21].

Peroxisome proliferator-activated receptor $\gamma$ (PPAR $\gamma$ ) agonists are a class of drugs that act as insulin sensitizers, providing glycemic control. Agonists of the related $\alpha$-subtype of the peroxisome proliferatoractivated receptor (PPAR $\alpha$ ) improve the lipid profile, lowering levels of TGs and raising levels of HDL-C. An ideal dual PPAR $\alpha / \gamma$ agonist would provide both glycemic control and an improved lipid profile at a well-tolerated therapeutic dose. Aleglitazar is a new, balanced dual PPAR $\alpha / \gamma$ agonist designed to optimize glycemic and lipid benefits, and minimize PPARrelated weight gain and edema in patients with T2DM. In vitro binding and transactivation assays have shown that aleglitazar is a potent and high-affinity ligand for both PPAR $\alpha\left(\mathrm{IC}_{50}=0.0028 \mu \mathrm{M}\right)$ and PPAR $\gamma\left(\mathrm{IC}_{50}=\right.$ $0.0046 \mu \mathrm{M})$ [22]. The balanced affinity of aleglitazar for both these receptors provides a dual PPAR $\alpha / \gamma$ agonist with therapeutically desirable characteristics and an improved safety profile [23], and distinguishes it from previously investigated imbalanced dual PPAR agonists that have been linked to adverse effects such as edema and renal complications [24,25].

The aim of this study was to assess the in vivo effects of aleglitazar on both glucose and lipid regulation in prediabetic rhesus monkeys.

\section{Methods}

\section{Subjects}

Six adult male rhesus monkeys with metabolic syndrome, were included in this study, based on meeting $\geq 3$ of the following criteria: waist circumference $>40 \mathrm{~cm}$ or waist/hip ratio $>0.9$, fasting plasma glucose $>72 \mathrm{mg} / \mathrm{dL}$, TG $>71 \mathrm{mg} / \mathrm{dL}, \mathrm{HDL}-\mathrm{C}<60 \mathrm{mg} / \mathrm{dL}$, and blood pressure: systolic $>130 \mathrm{mmHg}$ and/or diastolic $>80 \mathrm{mmHg}$. This definition parallels the criteria for the diagnosis of metabolic syndrome in humans according to the Adult Treatment Panel III guidelines [26]. The animals were individually housed and cared for according to the Guide for the Care and Use of Laboratory Animals [27]. Animals had ad libitum access to food (monkey chow: Lab Diet 5047; Purina Mills, St Louis, MO, USA) and water, and were maintained at an ambient temperature of $20^{\circ} \mathrm{C}$ to $24^{\circ} \mathrm{C}$ with a 12 -hour on-off lighting cycle. Primates were maintained in a facility approved by the Association for the Assessment and Accreditation of Laboratory and Animal Care (AAALAC) and the protocol was approved by the Institutional Animal Care and Use Committee.

\section{Study design}

Prior to initiation of the efficacy study, a 14-day pharmacokinetic (PK) assessment of a single dose of aleglitazar at $0.1 \mathrm{mg} / \mathrm{kg}$ was carried out in the same six animals; this consisted of vehicle administration (a piece of fruit $\sim 5 \mathrm{~g}$ ) for 7 days, followed by PK assessment of a single dose of orally administered aleglitazar $(0.1 \mathrm{mg} / \mathrm{kg})$ (results not shown), and a 4-day washout period. The efficacy study in the monkeys was initiated on day 15 and lasted for a total of 135 days. The efficacy study began on day 15 with a 28 -day baseline assessment phase for all six monkeys (vehicle only). Daily dosing with aleglitazar was started on day 44 and continued at a dose of $0.03 \mathrm{mg} / \mathrm{kg}$ per day for a period of $\sim 42$ days to day $\sim 86$, a 6 -week dosing period. This was followed by 63 -day washout period with vehicle (fruit). A schematic overview of the study design is given in Figure 1. Following this washout period, a lower dose of aleglitazar $(0.003 \mathrm{mg} / \mathrm{kg}$ per day) was assessed for a second 42-day period; however, as the majority of parameters did not return to baseline during the washout period, the results of this lower dose of aleglitazar are not presented.

In addition to standard serum chemistry and hematological assessments, blood samples were used to assess circulating levels of glucose, hemoglobin $\mathrm{A}_{1 \mathrm{c}}\left(\mathrm{HbA}_{1 \mathrm{c}}\right)$, insulin, lipids, and adiponectin (a surrogate marker of insulin sensitivity). For the high-dose efficacy study, blood samples ( $2 \mathrm{~mL}$ each) were collected on day 2 (prePK baseline), day 41 to day 43 (end of vehicle baseline), after 23 days of dosing, and at the end of the 42-day dosing period. Additional blood samples were collected periodically during the washout period $(14,28,42,54$, and 63 days after the last dose of aleglitazar).

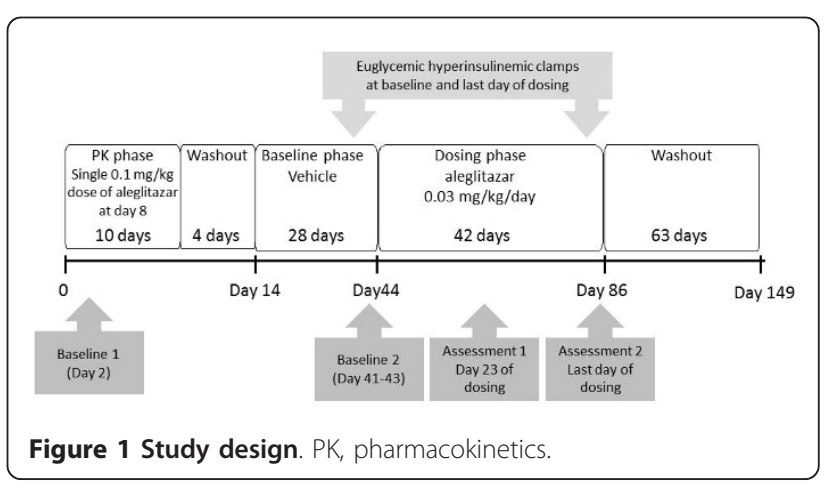


Insulin sensitivity was determined by a euglycemic hyperinsulinemic clamp procedure performed immediately prior to the initiation of dosing with aleglitazar and immediately after completion of the 42-day dosing period, with a target clamp glucose level of $85 \mathrm{mg} / \mathrm{dL}$ and a maximum stimulated insulin level of $>3000 \mu \mathrm{U} / \mathrm{mL}$ [4]. Electrocardiography, blood pressure, and heart rate were assessed during the pre-PK baseline, immediately prior to the initiation of aleglitazar, and after 42 days of dosing. Daily food intake (24-hour biscuit count) and biweekly body weight were recorded throughout the study.

\section{Assay techniques}

Venous blood samples (2 $\mathrm{mL})$ were drawn under light sedation (ketamine hydrochloride $10-15 \mathrm{mg} / \mathrm{kg}$ ) for blood chemistry and hematology. Blood was collected for serum, and also into a vacutainer tube containing EDTA for preparation of plasma. Fasting plasma glucose (FPG) was determined using the glucose oxidase method. Fasting plasma insulin (IRI) was evaluated at LINCO Diagnostic Services (St Charles, MO, USA) using a radioimmunoassay procedure. Hematologic and blood chemistry assays were performed by Anilab, Inc (Millstone Township, NJ, USA), while the $\mathrm{HbA}_{1 \mathrm{C}}$ level was obtained in-house using a DCA 2000+ analyzer (Bayer Diagnostics, Elkhart, IN, USA).

Total TG, HDL-C, LDL-C, and very low-density lipoprotein cholesterol (VLDL-C) were assayed by Penn Medical Laboratories (Hyattsville, MD, USA) from fresh plasma samples that had been fractionated by ultracentrifugation. In addition to the TG values as part of the basic metabolic panel, TG and lipoprotein constituents were also determined using two additional procedures to profile lipoprotein subclass distributions: (1) $\beta$ quantification (Penn Medical Laboratories) and (2) nuclear magnetic resonance (NMR) spectroscopy (LipoScience, Raleigh, NC, USA). The NMR spectroscopy lipoprotein subclass profiling technique uses the EDTA plasma from blood samples stored at $-80^{\circ} \mathrm{C}$ $[28,29]$ and provides a direct measure of the concentration of a specific lipoprotein subclass.

\section{Statistical analysis}

For comparison, the baseline for the high-dose efficacy assessment was defined as the mean of the baseline values obtained at day 2 (prior to the PK study) and at days 41 to 43 (prior to dosing with aleglitazar $0.03 \mathrm{mg} / \mathrm{kg}$ ), and the follow-up values were defined as the values obtained on the last day of dosing with $0.03 \mathrm{mg} / \mathrm{kg}$ aleglitazar.

Baseline and follow-up data were compared using Student's paired $t$-test. Whenever appropriate, nonparametric tests were performed. Values are expressed as mean \pm standard error and a significance level of $\mathrm{P}=$ 0.05 was used throughout this study. The baseline and follow-up data of plasma TG concentration were normalized by expressing on a natural logarithmic scale in order to compensate for the wide and non-normally distributed plasma TG concentrations.

\section{Results}

\section{Glycemic regulation}

Aleglitazar $0.03 \mathrm{mg} / \mathrm{kg}$ per day improved insulin sensitivity from baseline levels, as assessed by both euglycemic hyperinsulinemic clamp and adiponectin levels (a surrogate marker of insulin sensitivity). After 42 days of dosing, the mean glucose disposal rate (M-rate) increased from 7.8 to $12.5 \mathrm{mg} / \mathrm{kg}$ fat-free mass (FFM)/ minute; representing a $60 \%$ increase from baseline levels $(\mathrm{P}=0.001$; Figure $2 \mathrm{~A})$. Similarly, the mean levels of adiponectin rose by $158 \%$ from $12.8 \mu \mathrm{g} / \mathrm{mL}$ at baseline to $33.0 \mu \mathrm{g} / \mathrm{mL}(\mathrm{P}=0.003$; Figure $2 \mathrm{~B})$. Furthermore, circulating levels of insulin declined by $58 \%$ (146 to $61.2 \mu \mathrm{U} /$ $\mathrm{mL})$ after 42 days of dosing with aleglitazar $(\mathrm{P}=0.042$; Figure 2C).

Aleglitazar reduced FPG levels by $15 \%$ overall (89.0 to $75.3 \mathrm{mg} / \mathrm{dL} ; \mathrm{P}=0.161$; Figure 2D). In the subgroup of three monkeys with more elevated starting glucose levels $(105.8 \pm 2.0 \mathrm{mg} / \mathrm{dL})$, the glucose-lowering effect was enhanced resulting in a $28 \%$ decline to $76.2 \mathrm{mg} / \mathrm{dL}$. Note that normal lean rhesus monkeys have FPG levels of 60 to $75 \mathrm{mg} / \mathrm{dL}$, about $20 \mathrm{mg} / \mathrm{dL}$ lower than those of humans. FPG levels in these three hyperglycemic monkeys did not return to hyperglycemic levels following the 63-day washout period. No episodes of hypoglycemia were observed during treatment with aleglitazar. Mean levels of $\mathrm{HbA}_{1 \mathrm{c}}$ were non-significantly reduced from $6.7 \%$ to $5.6 \%$ after 42 days of aleglitazar treatment $(\mathrm{P}=0.092)$, and remained low during the subsequent washout period.

\section{Lipid regulation}

Aleglitazar decreased TG levels in all monkeys by an average of $89 \%$ (328 to $36 \mathrm{mg} / \mathrm{dL}$; $\mathrm{P}=0.057$ ) with $0.03 \mathrm{mg} / \mathrm{kg}$ per day (Figure 3A). Upon statistical normalization of the TG levels using logarithmic transformation, due to the wide baseline range (Figure 3A), a highly significant decrease was observed following administration of aleglitazar (Figure 3B; $\mathrm{P}=0.0048$ ). Total HDL-C levels increased by $125 \%$ (46 to 102 $\mathrm{mg} / \mathrm{dL})$ with aleglitazar $(\mathrm{P}=0.0007$; Figure $3 \mathrm{C})$. LDL$\mathrm{C}$ concentrations declined significantly by $41 \%$ with aleglitazar (92 to $54 \mathrm{mg} / \mathrm{dL} ; \mathrm{P}=0.015$; Figure $4 \mathrm{~A}$ ). Treatment with aleglitazar slightly increased the mean levels of apolipoprotein A-I (17\% increase; P = 0.096; Figure 3D) and apolipoprotein A-II (17\% increase; $\mathrm{P}=0.111)$. 
A

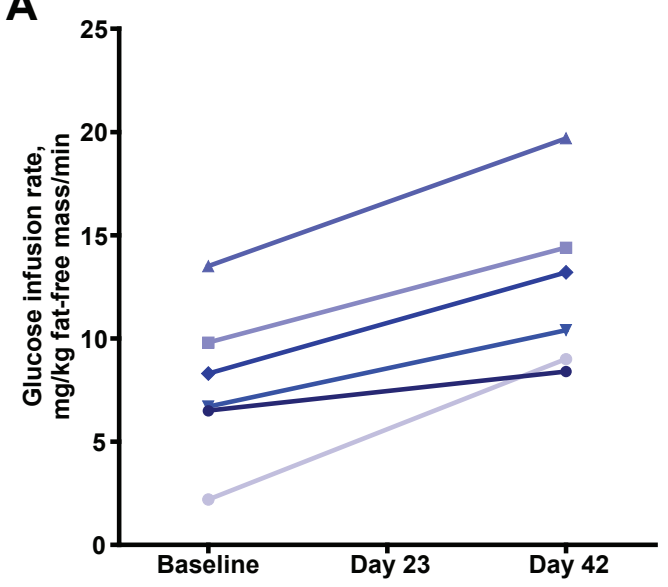

C

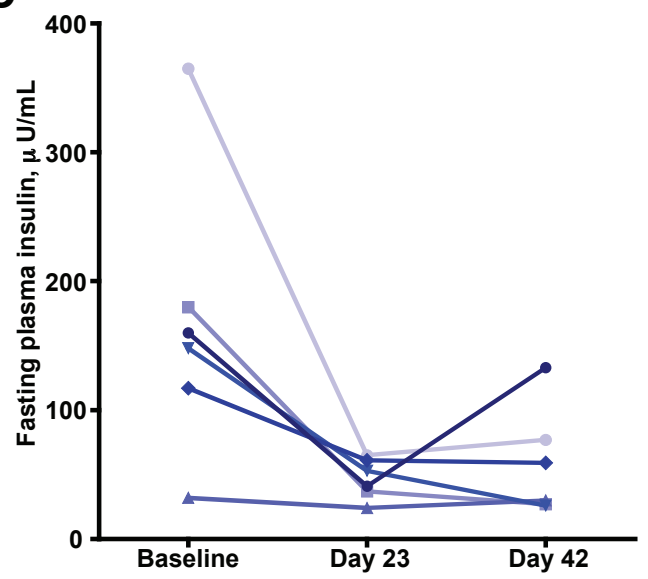

B

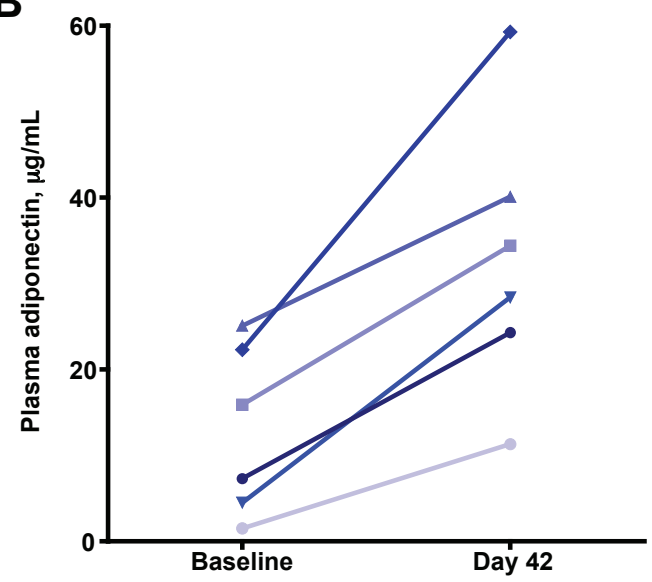

D

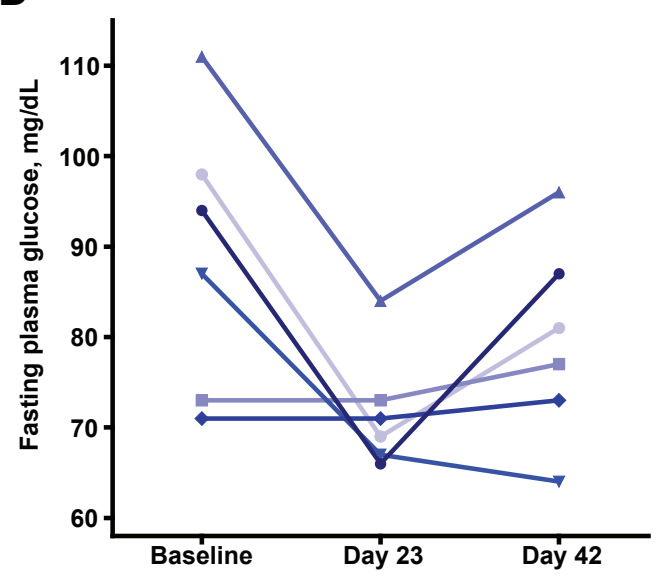

Figure 2 Individual levels of A) glucose disposal rate, B) adiponectin, C) fasting plasma insulin, and D) fasting plasma glucose in monkeys given aleglitazar $\mathbf{0 . 0 3} \mathbf{~ m g / k g}$ per day. The final day of dosing at $0.03 \mathrm{mg} / \mathrm{kg}$ per day aleglitazar (labeled in the figures as day 42), due to scheduling needs, ranged across monkeys from 44 to 46 days.

The NMR analysis of blood samples also revealed an $80 \%$ decrease in levels of the LDL2 (intermediate LDL - $26.6 \mathrm{~nm}$ diameter $)$ fraction $(\mathrm{P}=0.019)$ and a nonsignificant $30 \%$ increase in levels of the LDL1 (small dense LDL - $26 \mathrm{~nm}$ diameter) fraction $(\mathrm{P}=0.816)$ following administration of aleglitazar. The mean level of the LDL3 fraction (large buoyant LDL - $27 \mathrm{~nm}$ diameter) was non-significantly increased by $12 \%(\mathrm{P}=$ 0.831). Similarly, the mean level of apolipoprotein B dropped significantly by $57 \%(P=0.035$; Figure 4B) and levels of apolipoprotein C-III were non-significantly reduced by $36 \%$ as shown in Figure $4 \mathrm{D}(\mathrm{P}=$ 0.055) for all monkeys (not normalized). Additionally, mean VLDL-C levels decreased significantly by $93 \%$ $(P=0.041$; Figure $4 C)$ and this decrease was seen for all monkeys. The largest decreases in these lipid constituents were seen in those monkeys with the highest initial baseline levels.

\section{Food intake and body weight}

After 42 days of dosing with aleglitazar $0.03 \mathrm{mg} / \mathrm{kg}$ per day, mean body weight decreased significantly by about $5.9 \%$ from baseline $(P=0.043)$. A significant $32 \%$ decline in daily food intake $(\mathrm{P}=0.0097)$ was observed during administration of aleglitazar.

\section{Safety}

After 42 days of dosing with aleglitazar, mean blood urea nitrogen $(\mathrm{BUN}) /$ creatinine ratios increased slightly from a baseline value of 13.3 to $15.4(\mathrm{P}=0.235)$. All biochemical and hematological parameters were, and remained, within physiologically acceptable ranges, and no evidence of peripheral edema was observed. No adverse events or episodes of hypoglycemia were observed during the study.

No changes in heart rate were observed between baseline and the end of the aleglitazar dosing period, 
A

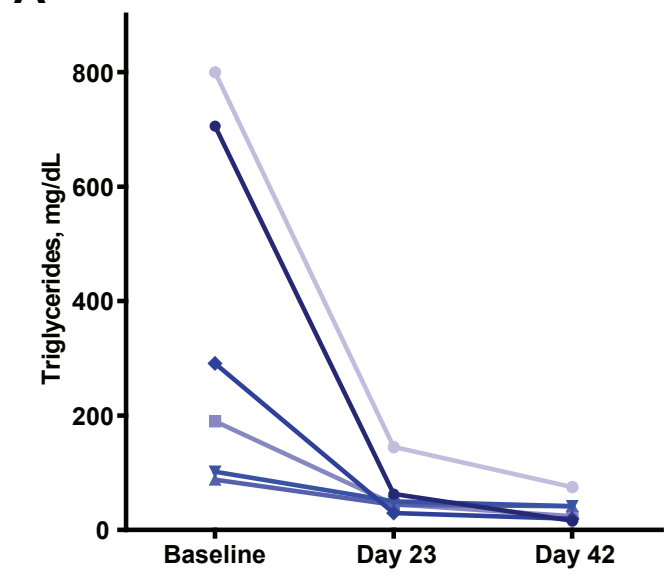

C

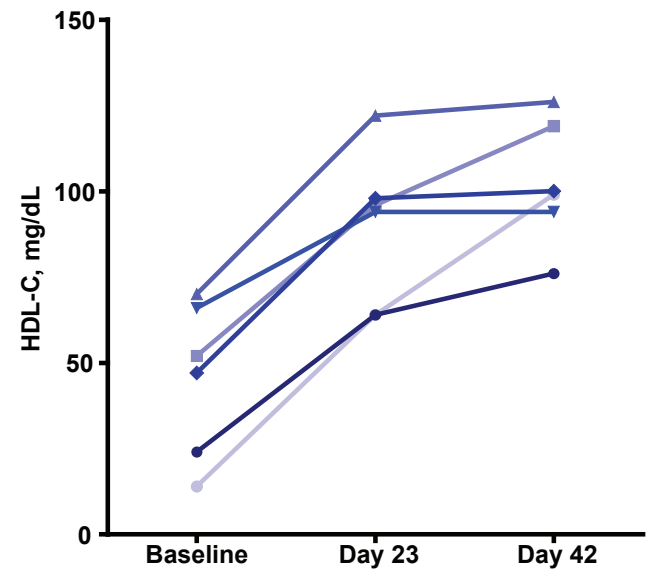

B

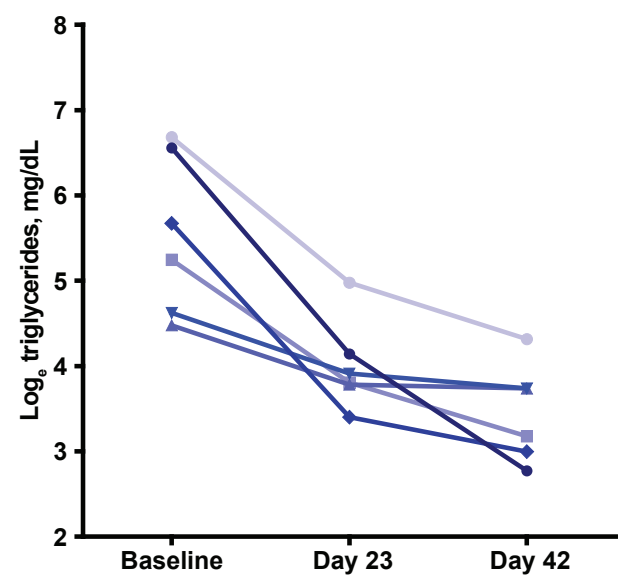

D

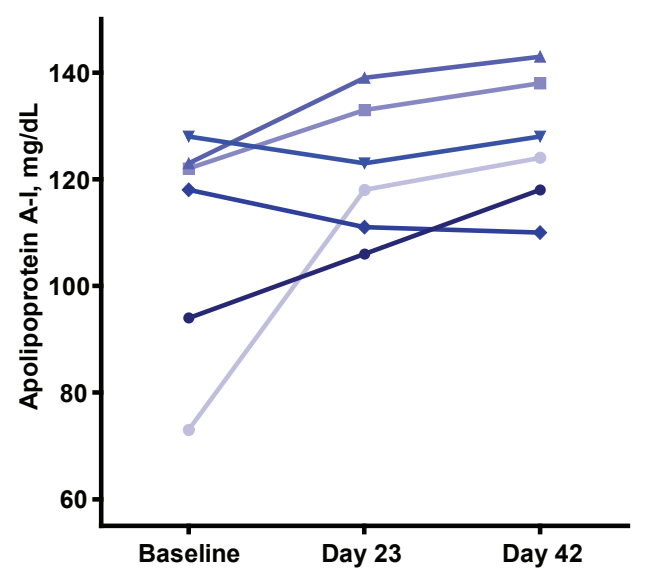

Figure 3 Individual levels of a) triglyceride, b) triglyceride (normalized), c) high-density lipoprotein cholesterol, and d) apolipoprotein A-I in monkeys given aleglitazar $\mathbf{0 . 0 3} \mathbf{~ m g} / \mathbf{k g}$ per day. The final day of dosing at $0.03 \mathbf{~ m g} / \mathbf{k g}$ per day aleglitazar (labeled in the figures as day 42), due to scheduling needs, ranged across monkeys from 44 to 46 days.

although non-significant reductions in systolic and diastolic blood pressure $(-13 \% ; \mathrm{P}=0.129$ and $-15 \%$; $\mathrm{P}=0.187$, respectively) were noted. Echocardiographic analysis revealed a pronounced, consistent, and significant $(\mathrm{P}=0.0036)$ decrease in total peripheral resistance (which was not compensated by a reflex tachycardia), and a trend to increased cardiac output $(\mathrm{P}=0.079)$ and stroke volume $(\mathrm{P}=0.227)$ between baseline and the end of aleglitazar dosing. All other parameters of cardiac function that were assessed showed no relevant changes. These data are consistent with a mild hypotensive and vasodilatory effect. There was no evidence of any deleterious effect on cardiac function.

\section{Discussion}

The results of this study in prediabetic rhesus monkeys demonstrate that the dual PPAR $\alpha / \gamma$ agonist aleglitazar has marked beneficial effects on $\mathrm{HbA}_{1 \mathrm{c}}$, FPG, insulin sensitivity, TGs, LDL-C, HDL-C, and other lipid parameters.
Laboratory and physiological assessments indicated that aleglitazar was well tolerated at $0.03 \mathrm{mg} / \mathrm{kg}$ per day.

The results indicated an overall beneficial effect of aleglitazar on cholesterol regulation in the monkeys. With aleglitazar, fasting TG and LDL-C levels were significantly reduced, while HDL-C level was significantly increased by $125 \%$. The effects of these improvements in the lipid profile of the monkeys are difficult to extrapolate to disease progression, as a wide spectrum of lipids contributes to a varying degree to the development of cardiovascular disease or diabetes [28,30,31]. However, together with the modifications in the plasma concentrations of all classes of apolipoprotein following the administration of aleglitazar, as well as an apparent change from a profile of small dense LDL to larger LDL particles, this represents a clear shift to a less atherogenic profile in the obese monkeys and may indicate that progression of cardiovascular disease would be delayed by long-term treatment with this agonist. 
A

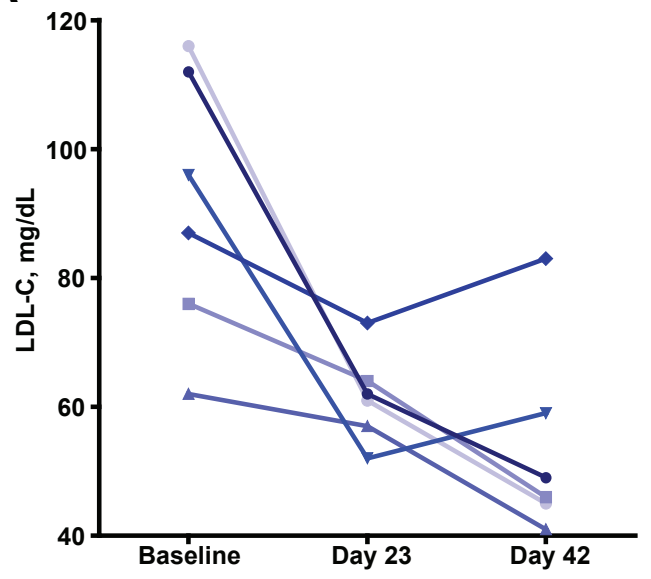

C

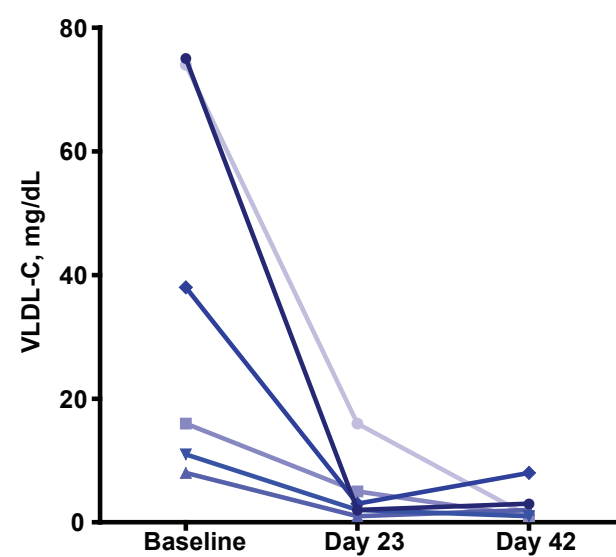

B

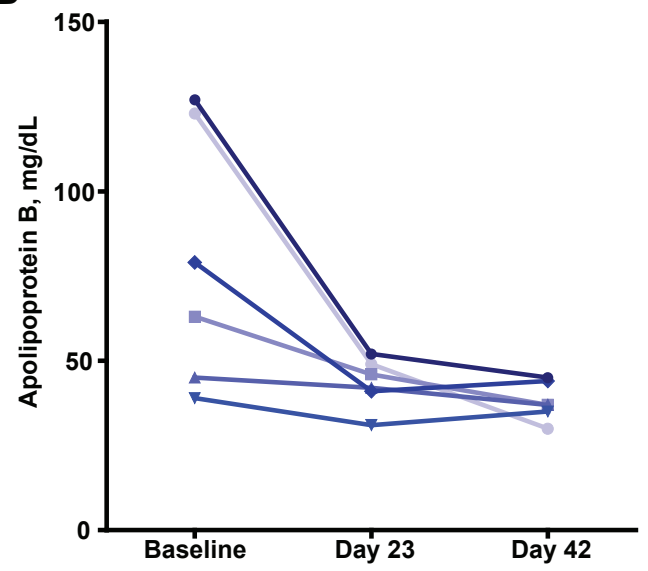

D

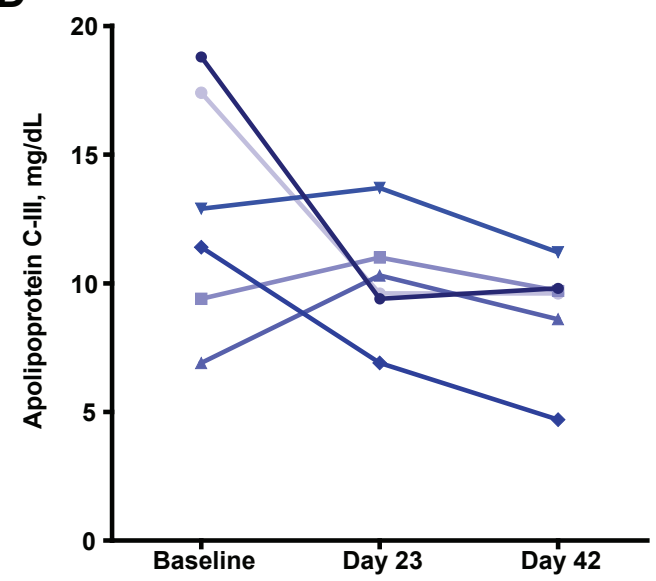

Figure 4 Individual levels of A) low-density lipoprotein cholesterol, B) apolipoprotein B, C) very low-density lipoprotein cholesterol, and D) apolipoprotein C-III in monkeys given aleglitazar $0.03 \mathbf{~ m g} / \mathbf{k g}$ per day. The final day of dosing at $0.03 \mathrm{mg} / \mathrm{kg}$ per day aleglitazar (labeled in the figures as day 42), due to scheduling needs, ranged across monkeys from 44 to 46 days.

In addition to the lipid benefits observed with aleglitazar, food intake and body weight were reduced during administration of the study drug. A pronounced decrease in circulating levels of TG might result from reduced food intake, and consequently, decreased body weight, as seen in animals treated with this dose of aleglitazar. This differentiates aleglitazar from PPAR $\gamma$ agonists that are associated with increases in body weight. The mechanism explaining these findings is not clear, but PPAR $\alpha$-mediated effects of the compound on adipose tissue (e.g., increased lipolysis and/or fatty acid oxidation) cannot be excluded. Phase II clinical trial data in patients with T2DM demonstrated that body weight increased to a lesser extent with aleglitazar, when compared with pioglitazone in a head-to-head comparison study [32]. Although the direct comparison between aleglitazar and pioglitazone was not performed in our study, previous thiazolidinediones studies in rhesus monkeys [15,33] support the notion that gains in body weight would be less evident with aleglitazar.

Beneficial effects of aleglitazar were also evident in improved glycemic control. Administration of aleglitazar not only reduced FPG, fasting insulin, and $\mathrm{HbA}_{1 \mathrm{c}}$ levels in the monkeys, but also improved insulin sensitivity, as measured by the euglycemic hyperinsulinemic clamp. The $60 \%$ increase in glucose infusion rate following administration of aleglitazar was in accordance with the observed increase in plasma adiponectin, a surrogate marker of insulin sensitivity.

The observations that aleglitazar improved glucose and lipid regulation in a non-human primate model of the metabolic syndrome and impaired glucose tolerance (prediabetes), are largely consistent with previously published studies of aleglitazar in other animal models including Zucker Diabetic Fatty (ZDF) rats and human ApoAI-transgenic mice [22]. The beneficial effects of 
aleglitazar on the lipid and glycemic profiles of the obese monkeys in this study, especially the increase of HDL-C levels, are consistent with other dual PPAR agonists previously tested in a rhesus monkey model, such as TAK-559 [14]. Ding et al. demonstrated that TAK559 treatment resulted in significant elevation of HDL$\mathrm{C}$, increased apolipoprotein A-I, with concomitant decreases in plasma TG and apolipoprotein B. It is believed that enhanced reverse cholesterol transport via HDL may be involved in the improved lipid profile in monkeys administered dual PPAR agonists, such as aleglitazar and TAK-559 [34]. However, unlike aleglitazar, TAK-559 did not elicit significant changes in the levels of VLDL-C or LDL-C.

Selective PPAR $\gamma$ agonists, such as thiazolidinediones, elicit improvements in insulin sensitivity and glucose tolerance, but are often associated with weight gain and edema in humans [35]. Selective PPAR $\alpha$ agonists, such as fibrates, are associated with improvements in the lipid profile, but are also associated with increases in serum creatinine, with minimal effect on glycemia. Compounds that act on both receptors may represent an attractive treatment option, provided that the potential to improve both glycemic and lipid parameters can be achieved within the same therapeutic window in order to minimize the incidence of PPAR-related side effects. In this study there were no safety concerns following administration of either dose of aleglitazar, with no cases of hypoglycemia, edema, or body weight gain. The development of previous dual PPAR agonists has been halted by safety concerns; however, the safety profile of aleglitazar is encouraging [36].

The interpretation of relevance and the extrapolation of the results of the present study to human subjects must be undertaken with knowledge of its limitations that include: the subjects studied were not humans, and dose concordance between humans and monkeys is unknown.

\section{Conclusions}

Given the close similarity between the rhesus monkey model used in this study and the diabetic state in humans, these findings suggest that aleglitazar, a dual PPAR $\alpha / \gamma$ agonist, has beneficial effects on both lipid and glucose parameters and may have a therapeutic role in modifying cardiovascular risk factors and improving glycemic control in patients with T2DM.

\section{List of abbreviations}

BUN: blood urea nitrogen; FFM: fat-free mass; FPG: fasting plasma glucose; $\mathrm{HbA}_{1 c}$ : hemoglobin $\mathrm{A}_{1 c}$; $\mathrm{HDL}-\mathrm{C}$ : high-density lipoprotein cholesterol; IRI: plasma insulin; LDL-C: low-density lipoprotein cholesterol; NMR: nuclear magnetic resonance; PK: pharmacokinetics; PPAR: peroxisome proliferatoractivated receptor; T2DM: type 2 diabetes mellitus; TG: triglyceride; VLDL-C: very low-density lipoprotein cholesterol.

\section{Acknowledgements}

Editorial assistance in preparation of the report for submission was provided by Paul Lane, PhD, of Evidence Scientific Solutions Ltd., Horsham, UK, and was funded by F Hoffmann-La Roche AG.

Funding sources

This study was funded by F. Hoffmann-La Roche AG.

\section{Author details}

${ }^{1}$ Department of Internal Medicine and Pediatrics, University of South Florida, Tampa, FL, USA. ²Department of Molecular Pharmacology and Physiology, University of South Florida, Tampa, FL, USA. ${ }^{3}$ F. Hoffmann-La Roche AG, Basel, Switzerland.

\section{Authors' contributions}

All authors contributed to the design, analysis, and interpretation of this study. BH and XT: performed the Rhesus monkey studies. All authors contributed to the writing of this manuscript and have approved this version for submission.

\section{Competing interests}

Agnes Bénardeau, Markus Meyer, Elena Sebokova, and Jacques Mizrahi are employees of F. Hoffmann-La Roche AG. Dr. Tigno's work on this manuscript was performed during her tenure at the University of South Florida and does not reflect the view of the NIH or the United States government.

Received: 23 December 2010 Accepted: 20 January 2011

Published: 20 January 2011

\section{References}

1. Fact Sheet No. 312 - Diabetes. [http://www.who.int/mediacentre/ factsheets/fs312/en/]

2. American Diabetes Association: Standards of medical care in diabetes2008. Diabetes Care 2008, 31(Suppl 1):S12-S54

3. Graham I, Atar D, Borch-Johnsen K, Boysen G, Burell G, Cifkova R, Dallongeville J, De Backer G, Ebrahim S, Gjelsvik B, Herrmann-Lingen C, Hoes A, Humphries S, Knapton M, Perk J, Priori SG, Pyorala K, Reiner Z, Ruilope L, Sans-Menendez S, Scholte op Reimer W, Weissberg P, Wood D, Yarnell J, Zamorano JL, Walma E, Fitzgerald T, Cooney MT, Dudina A, Vahanian A, Camm J, De Caterina R, Dean V, Dickstein K, Funck-Brentano C, Filippatos G, Hellemans I, Kristensen SD, McGregor K, Sechtem U, Silber S, Tendera M, Widimsky P, Zamorano JL, Hellemans I, Altiner A, Bonora E, Durrington PN, Fagard R, Giampaoli S, Hemingway H, Hakansson J, Kjeldsen SE, Larsen ML, Mancia G, Manolis AJ, Orth-Gomer K, Pedersen T, Rayner M, Ryden L, Sammut M, Schneiderman N, Stalenhoef AF, Tokgözoglu L, Wiklund O, Zampelas A: European guidelines on cardiovascular disease prevention in clinical practice: executive summary. Eur Heart J 2007, 28:2375-2414.

4. Bodkin NL, Metzger BL, Hansen BC: Hepatic glucose production and insulin sensitivity preceding diabetes in monkeys. Am J Physiol 1989, 256: E676-E681.

5. Clark A, de Koning EJ, Hattersley AT, Hansen BC, Yajnik CS, Poulton J: Pancreatic pathology in non-insulin dependent diabetes (NIDDM). Diabetes Res Clin Pract 1995, 28(Suppl):S39-S47.

6. Hansen $B C$, Bodkin NL: Heterogeneity of insulin responses: phases leading to type 2 (non-insulin-dependent) diabetes mellitus in the rhesus monkey. Diabetologia 1986, 29:713-719.

7. Hansen BC, Bodkin NL: Beta-cell hyperresponsiveness: earliest event in development of diabetes in monkeys. Am J Physiol 1990, 259: R612-R617.

8. Hansen BC, Striffler JS, Bodkin NL: Decreased hepatic insulin extraction precedes overt noninsulin dependent (type II) diabetes in obese monkeys. Obes Res 1993, 1:252-260.

9. Hansen BC: Primate models of type 2 diabetes. In Diabetes Mellitus: A Fundamental and Clinical Text. 3 edition. Edited by: LeRoith D, Olefsky JM, Taylor SI. Philadelphia: Lippincott, Williams 2004:1059-1074.

10. Tigno XT, Hansen BC: The rhesus monkey (Macaca mulatta) manifests all features of human type 2 diabetes. In Animal Models of Diabetes: Frontiers in Research. 2 edition. Edited by: Shafrir E. New York: CRC Press; 2007:251-270 [http://www.crcpress.com/product/isbn/9780849395345].

11. Hansen BC: Chronomics of the metabolic syndrome. In The Metabolic Syndrome: Epidemiology, Clinical Treatment, and Underlying Mechanisms. 
Edited by: Hansen BC, Bray GA. Totowa, New Jersey: Humana Press; 2008:373-386.

12. Ding SY, Tigno XT, Hansen BC: Nuclear magnetic resonance-determined lipoprotein abnormalities in nonhuman primates with the metabolic syndrome and type 2 diabetes mellitus. Metabolism 2007, 56:838-846.

13. Bodkin NL, Alexander TM, Ortmeyer HK, Johnson E, Hansen BC: Mortality and Morbidity in laboratory-maintained rhesus monkeys and effects of long-term dietary restriction. J Gerontol A Biol Sci 2003, 58(3):212-219.

14. Ding SY, Tigno XT, Braileanu GT, Ito K, Hansen BC: A novel peroxisome proliferator-activated receptor $\alpha / \gamma$ dual agonist ameliorates dyslipidemia and insulin resistance in prediabetic rhesus monkeys. Metabolism 2007, 56:1334-1339

15. Kemnitz JW, Elson DF, Roecker EB, Baum ST, Bergman RN, Meglasson MD: Pioglitazone increases insulin sensitivity, reduces blood glucose, insulin, and lipid levels, and lowers blood pressure, in obese, insulin-resistant rhesus monkeys. Diabetes 1994, 43:204-211.

16. Kharitonenkov A, Wroblewski VJ, Koester A, Chen YF, Clutinger CK, Tigno XT, Hansen BC, Shanafelt AB, Etgen GJ: The metabolic state of diabetic monkeys is regulated by fibroblast growth factor-21. Endocrinology 2007, 148:774-781

17. Oliver WR Jr, Shenk JL, Snaith MR, Russell CS, Plunket KD, Bodkin NL, Lewis MC, Winegar DA, Sznaidman ML, Lambert MH, Xu HE, Sternbach DD, Kliewer SA, Hansen BC, Willson TM: A selective peroxisome proliferatoractivated receptor $\delta$ agonist promotes reverse cholesterol transport. Proc Natl Acad Sci USA 2001, 98:5306-5311.

18. Winegar DA, Brown PJ, Wilkison WO, Lewis MC, Ott RJ, Tong WQ, Brown HR, Lehmann JM, Kliewer SA, Plunket KD, Way JM, Bodkin NL, Hansen BC: Effects of fenofibrate on lipid parameters in obese rhesus monkeys. J Lipid Res 2001, 42:1543-1551.

19. Ortmeyer HK, Adall Y, Marciani KR, Katsiaras A, Ryan AS, Bodkin NL, Hansen BC: Skeletal muscle glycogen synthase subcellular localization: effects of insulin and PPAR- $a$ agonist (K-111) administration in rhesus monkeys. Am J Physiol Regul Integr Comp Physiol 2005, 288:R1509-R1517.

20. Schäfer SA, Hansen BC, Völkl A, Fahimi HD, Pill J: Biochemical and morphological effects of K-111, a peroxisome proliferator-activated receptor (PPAR)a activator, in non-human primates. Biochem Pharmacol 2004, 68:239-251.

21. Bodkin NL, Pill J, Meyer K, Hansen BC: The effects of K-111, a new insulinsensitizer, on metabolic syndrome in obese prediabetic rhesus monkeys. Horm Metab Res 2003, 35:617-624.

22. Bénardeau $A$, Benz J, Binggeli A, Blum D, Boehringer M, Grether $U$, Hilpert H, Kuhn B, Märki HP, Meyer M, Püntener K, Raab S, Ruf A, Schlatter D, Mohr P: Aleglitazar, a new, potent, and balanced dual PPARa/ $/$ agonist for the treatment of type II diabetes. Bioorg Med Chem Lett 2009, 19:2468-2473.

23. Pourcet B, Fruchart JC, Staels B, Glineur C: Selective PPAR modulators, dual and pan PPAR agonists: multimodal drugs for the treatment of type 2 diabetes and atherosclerosis. Expert Opin Emerg Drugs 2006, 11:379-401.

24. Nissen SE, Wolski K, Topol EJ: Effect of muraglitazar on death and major adverse cardiovascular events in patients with type 2 diabetes mellitus. JAMA 2005, 294:2581-2586.

25. Balakumar P, Rose M, Ganti SS, Krishan P, Singh M: PPAR dual agonists: are they opening Pandora's Box? Pharmacol Res 2007, 56:91-98.

26. Grundy SM, Cleeman II, Daniels SR, Donato KA, Eckel RH, Franklin BA, Gordon DJ, Krauss RM, Savage PJ, Smith SC Jr, Spertus JA, Costa F: Diagnosis and management of the metabolic syndrome: an American Heart Association/National Heart, Lung, and Blood Institute Scientific Statement. Circulation 2005, 112:2735-2752.

27. Institute of Laboratory Animal Research, Commission on Life Sciences, National Research Council: Guide for the Care and Use of Laboratory Animals Washington, DC National Academy Press; 1996.

28. Otvos JD: Measurement of lipoprotein subclass profiles by nuclear magnetic resonance spectroscopy. Clin Lab 2002, 48:171-180.

29. Otvos JD, Jeyarajah EJ, Bennett DW, Krauss RM: Development of a proton nuclear magnetic resonance spectroscopic method for determining plasma lipoprotein concentrations and subspecies distributions from a single, rapid measurement. Clin Chem 1992, 38:1632-1638.

30. Brunzell JD, Ayyobi AF: Dyslipidemia in the metabolic syndrome and type 2 diabetes mellitus. Am J Med 2003, 115(Suppl 8A):24S-28S.

31. Garvey WT, Kwon S, Zheng D, Shaughnessy S, Wallace P, Hutto A, Pugh K, Jenkins AJ, Klein RL, Liao Y: Effects of insulin resistance and type 2 diabetes on lipoprotein subclass particle size and concentration determined by nuclear magnetic resonance. Diabetes 2003, 52:453-462.

32. Henry RR, Lincoff AM, Mudaliar S, Rabbia M, Chognot C, Herz M: Effect of the dual peroxisome proliferator-activated receptor- $\alpha / \gamma$ agonist aleglitazar on risk of cardiovascular disease in patients with type 2 diabetes (SYNCHRONY): a phase II, randomised, dose-ranging study. Lancet 2009, 374:126-135.

33. Ortmeyer HK, Sajan MP, Miura A, Kanoh Y, Rivas J, Li Y, Standaert ML, Ryan A, Bodkin NL, Farese RV, Hansen BC: Insulin signaling and insulin sensitizing in muscle and liver of obese monkeys: PPARy agonist improves defective activation of atypical protein kinase C. Antioxid Redox Signal 2011, 14(2):207-19.

34. Wallace JM, Schwarz M, Coward P, Houze J, Sawyer JK, Kelley KL, Chai A, Rudel LL: Effects of peroxisome proliferator-activated receptor $\mathrm{a} / \delta$ agonists on HDL-cholesterol in vervet monkeys. J Lipid Res 2005, 46:1009-1016.

35. Fonseca V: Effect of thiazolidinediones on body weight in patients with diabetes mellitus. Am J Med 2003, 115(Suppl 8A):42S-48S

36. Fisman EZ, Tenenbaum A: A cardiologic approach to non-insulin antidiabetic pharmacotherapy in patients with heart disease. Cardiovas Diabetol 2009, 8:38.

doi:10.1186/1475-2840-10-7

Cite this article as: Hansen et al:: Effects of aleglitazar, a balanced dual peroxisome proliferator-activated receptor $\alpha / \gamma$ agonist on glycemic and lipid parameters in a primate model of the metabolic syndrome. Cardiovascular Diabetology 2011 10:7.

\section{Submit your next manuscript to BioMed Central and take full advantage of:}

- Convenient online submission

- Thorough peer review

- No space constraints or color figure charges

- Immediate publication on acceptance

- Inclusion in PubMed, CAS, Scopus and Google Scholar

- Research which is freely available for redistribution

Submit your manuscript at www.biomedcentral.com/submit
Biomed Central 